\title{
Geriatric Anorexia Nervosa
}

\author{
Moaweya Zayed, MD, and Joseph P. Garry, MD
}

Eating disorders are not commonly diagnosed in individuals aged $>50$ years, yet they are associated with significant psychiatric comorbidities and overall morbidity. Anorexia nervosa is the most common eating disorder among this age group, and women are affected most often. We present the fatal case of a 66-year old woman with severe malnutrition and newly diagnosed anorexia nervosa. Inpatient refeeding was unsuccessful, and she succumbed to multisystem organ failure. The timely recognition of eating disorders among older people is important for family physicians who care for patients across the life spectrum. (J Am Board Fam Med 2017;30:666-669.)

Keywords: Anorexia Nervosa, Comorbidity, Family Physicians, Feeding and Eating Disorders, Inpatients, Morbidity, Protein-Energy Malnutrition

The onset of eating disorders has been most commonly described as occurring among adolescent and young adult women (16 to 25 years old). More controversy over the emergence of eating disorders in women aged $>50$ years has evolved regarding whether the eating disorder emerges at that time or is diagnosed from onset at an earlier age. ${ }^{1-3}$ The behaviors that coincide with eating disorders, such as restrictive eating patterns and body dysmorphia, have been documented among older women. Among a random community sample of 475 women aged 60 to 70 years, $3.8 \%$ met the criteria for an eating disorder. ${ }^{4}$ Among older Canadian women, $2.6 \%$ of those aged 50 to 64 years, and $1.8 \%$ of those aged $>65$, reported symptoms of disordered eating. ${ }^{5}$

A strong association has been noted with comorbid psychiatric disorders in late-onset eating disorders. Major depression has been shown to be 2.4 to 4 times more likely in individuals with anorexia

This article was externally peer reviewed.

Submitted 3 May 2017; revised 3 June 2017; accepted 8 June 2017.

From University of Minnesota Medical Center Family Medicine Residency Program, Minneapolis (MZ); and the Department of Family Medicine and Community Health, University of Minnesota, Minneapolis (JPG).

Current affiliation: Mayo Clinic Health System-Eastridge, Mankato, MN (MZ).

Funding: none.

Conflict of interest: none declared.

Corresponding author: Joseph P. Garry, MD, Department of Family Medicine and Community Health, 909 Fulton St. SE, Minneapolis, MN 55455 (E-mail: Garry036@umn.edu). than in controls. ${ }^{6}$ Anxiety disorders, obsessive compulsive disorder, and social phobias have also been associated with eating orders. ${ }^{7,8}$ In a study of women aged $\geq 50$ years that investigated the relationship between eating disorder symptomatology (scores $>20$ on the EAT-26 eating disorders screening tool, where scores $>20$ indicate high risk of an eating disorder) and mood disorders, older women with eating disorder symptomatology had a significantly higher risk of a mood disorder than did those women who scored $\leq 20$ on the EAT-26 module (odds ratio, 6.9 [95\% confidence interval, 4.5-10.4] and 1.0, respectively). ${ }^{2}$ A recent review of the literature regarding eating disorders in individuals aged $>50$ years revealed comorbid psychiatric conditions in $60 \%$ of this population; major depression was the most common mood disorder among patients in this age group. ${ }^{9}$

Anorexia nervosa (Table 1) is the most common eating disorder among older people. ${ }^{9}$ The mortality associated with late-onset eating disorders is considerable. A review of $>10$ million death records from the United States over a 4-year time period found an average of 145 deaths annually for which anorexia nervosa was the primary or contributing factor. ${ }^{10}$ This equates to an annual rate of 6.73 per 100,000 deaths in the United States. More specifically, in a review of eating disorders in those aged $>50$, mortality attributed to either the eating disorder or a complication of the eating disorder was $21 \%$. 
Table 1. Diagnostic Criteria for Anorexia Nervosa. From the Diagnostic and Statistical Manual of Mental Disorders $^{11}$

\begin{tabular}{l}
\hline Restriction of energy intake relative to requirements \\
leading to a significantly low body weight in the \\
context of age, sex, developmental trajectory, and \\
physical health. \\
Intense fear of gaining weight or becoming fat, even \\
though underweight. \\
Disturbance in the way in which one's body weight \\
or shape is experienced, undue influence of body \\
weight or shape on self-evaluation, or denial of \\
the seriousness of the current low body weight.
\end{tabular}

Here we present a case of an older woman presenting with severe malnutrition and undiagnosed anorexia nervosa.

\section{Case}

A married 66-year-old woman with 16 years of formal education was admitted to the university hospital for worsening weakness and shortness of breath over months that was now limiting her ability to ambulate. She also reported worsening abdominal discomfort and cramping and a general nonspecific reduction in appetite. She denied any other symptoms. Her history was significant for generalized anxiety, hypertension, peptic ulcer disease, irritable bowel syndrome, hypothyroidism, gastroesophageal reflux with esophagitis, and ongoing malnutrition (decrease in body weight and albumin concentrations $<3 \mathrm{~g} / \mathrm{dL}$ [range, 3.4-5.0 $\mathrm{g} / \mathrm{dL}$ ] since 2013). She had undergone a prior cholecystectomy and hysterectomy. A chart review demonstrated a weight of $59.4 \mathrm{~kg}$ in 2006 and then a dramatic change in weight to $46.2 \mathrm{~kg}$ in 2009 , which was associated with concerns about an eating disorder voiced by her spouse and adult children, and concerns from her primary physician upon continued unrevealing medical evaluations (2-item $\mathrm{Pa}$ tient Health Questionnaire scores revealed no depressive symptoms and negative results on all of the following: chest/abdomen/pelvic computed tomography, prior Papanicolaou tests per US Preventive Services Task Force guidelines, mammograms, fecal occult blood tests, colonoscopy, rectal biopsies, esophagogastroduodenoscopy, and gastric and smallbowel biopsies). The patient refused to consult with a psychologist or nutritionist. Her family also noted increasing social isolation on the part of the patient.

Her physical examination was remarkable for a height of $1.57 \mathrm{~m}$, weight of $43.5 \mathrm{~kg}$ (felt to be falsely elevated because of the presence of an estimated 3 to $4 \mathrm{~kg}$ of fluid resulting from third spacing), and a body mass index of $17.5 \mathrm{~kg} / \mathrm{m}^{2}$ (the chart review demonstrated that her body mass index had not exceeded $18.7 \mathrm{~kg} / \mathrm{m}^{2}$ in the prior 7 years for which data were available). She was cachectic in appearance, had peripheral edema of the upper and lower extremities, and had new pressure sores on her back and buttocks. Electrocardiography demonstrated low voltage but otherwise only nonspecific $\mathrm{T}$ wave changes. Chest radiography demonstrated moderate bilateral pleural effusions. Laboratory findings were significant for depressed levels of hematocrit at 31.8\% (35-47\%), glucose at $69 \mathrm{mg} / \mathrm{dL}(70-99 \mathrm{mg} / \mathrm{dL})$, calcium at 6.9 $\mathrm{mg} / \mathrm{dL}(8.5-10.1 \mathrm{mg} / \mathrm{dL})$, protein at $4.4 \mathrm{~g} / \mathrm{dL}(6.8-$ $8.8 \mathrm{~g} / \mathrm{dL})$, albumin at $1.4 \mathrm{~g} / \mathrm{dL}(3.4-5.0 \mathrm{~g} / \mathrm{dL})$, and prealbumin at $9 \mathrm{mg} / \mathrm{dL}(15-45 \mathrm{mg} / \mathrm{dL})$. She had an elevated brain natriuretic peptide concentration of $12,268 \mathrm{pg} / \mathrm{mL}(0-900 \mathrm{pg} / \mathrm{mL})$ and normal values for alanine aminotransferase at $16 \mathrm{U} / \mathrm{L}(0-50 \mathrm{U} / \mathrm{L})$, aspartate aminotransferase at $26 \mathrm{U} / \mathrm{L}(0-45 \mathrm{U} / \mathrm{L})$, and thyroid-stimulating hormone at $1.81 \mathrm{mU} / \mathrm{L}$ (0.40-4.00 mU/L). Her echocardiogram demonstrated an ejection fraction of $50 \%$ to $55 \%$, with mild valvular changes and moderate ascites. Pleural fluid analysis was transudative and negative for malignancy.

She was admitted, thoroughly evaluated, and prescribed enteral feedings with the support of nutrition services. A psychiatric evaluation reported a high likelihood of anorexia nervosa. When asked whether she looks in the mirror and thinks she is too thin, she responded with "I am what I am," but she was not willing to elaborate further. She did admit to energy restriction, fear of weight gain, and disturbance in the way she experiences her body weight. She expressed denial of the seriousness of her current body weight and malnutrition at presentation, thereby fulfilling diagnostic criteria for anorexia nervosa (Table 1).

The patient developed refeeding syndrome with her enteral feedings and marked shifts in electrolytes, as well as significant problems with third spacing of fluids, including increasing pleural effusions and ascites and worsening peripheral edema. She underwent multiple thoracenteses to remove the pleural fluid in order to assist with her respiratory status. Despite intensive medical and nutritional care, she developed multiorgan failure and died. 


\section{Discussion}

Anorexia nervosa can present initially in older adults, and women in this population are most commonly affected. ${ }^{1,12,13}$ A review of the literature on eating disorders in older people showed the mean age of patients to be 68.6 years, $88 \%$ of whom are women, and $81 \%$ of all cases of eating disorders were diagnosed as anorexia nervosa. ${ }^{9}$ It is also noteworthy that $69 \%$ of cases of anorexia nervosa in this population were late onset, as opposed to the more common early onset. Our case seemed to be more consistent with late-onset anorexia nervosa. Despite case reports and literature reviews of late-onset eating disorders, a controversy exists as to whether eating disorders can initially manifest later in life or are a recurrence of an early-onset eating disorder. ${ }^{1,9,12,13}$ Patient records were reviewed for individuals aged $>50$ years who were admitted to national eating disorders center over a period of 10 years in an attempt to clarify this issue of eating disorders that present late, but the review found no cases of late-onset eating disorders in that population. ${ }^{3}$ The possibility that eating disorders in older persons are neither well recognized nor diagnosed, or that older persons are more adept at disguising their eating disorder, should be considered. Regardless of this controversy regarding lateonset eating disorders, physicians can expect to encounter older patients with eating disorders and should be aware of the potential to diagnose anorexia nervosa in this population.

The comorbidity of mood disorders with eating disorders is well recognized. Among older individuals with eating disorders, a particular association exists with comorbid depression. ${ }^{9}$ A recent metaanalysis of the relationship of eating pathology and depression demonstrated that eating disorders and depression are concurrent risk factors for each other. ${ }^{14}$ This is an associative relationship between eating disorders and depression, and not a causal one. Older community-dwelling women have also been shown to have a marked increased risk for any mood disorder in association with aberrant dieting or oral control behaviors (eg, cutting food into small pieces, taking longer to eat than others, displaying self-control around food). ${ }^{2}$ Among older women with anorexia nervosa admitted to an eating disorder center, high levels of both psychiatric and physical comorbidities have been demonstrated. ${ }^{3}$ We noted a history of generalized anxiety in our patient, as well as her family's concern for increasing social isolation, both of which have been associated with eating disorders.

While few data are available on the management of older adults presenting with anorexia nervosa, the combination of both behavioral and pharmacologic treatment has been found to be the most successful. ${ }^{9}$ Caution is appropriate, however, as only $42 \%$ of patients reviewed were successfully managed with this treatment combination. Affected patients perceive the limited resources for the successful management of eating disorders in older patients, and this is a potential barrier to treatment. ${ }^{3}$ The challenge for physicians is to identify available resources for this unique population. Based on the review of our patient's chart, finding appropriate resources for referral may have been an issue of concern. This also raises questions regarding the diagnosis of anorexia nervosa in older patients, as physicians will certainly be concerned about other organic causes of weight loss, and it is possible that a diagnosis may be delayed or missed as a result of these concerns.

The medical complications of anorexia nervosa are numerous and well known: cardiac arrhythmia, heart failure, aspiration pneumonia, respiratory failure, pancytopenia, renal failure, hypoglycemia, hypercortisolism, thyroid abnormalities, osteoporosis, hepatitis, pancreatitis, constipation, and cerebral atrophy. Yet, few data are available on the overall morbidity and mortality of eating disorders among older adults. Anorexia nervosa was found to cause or significantly contribute to mortality in up to $21 \%$ of deaths in older adults with the diagnosis. ${ }^{9}$ This suggests that anorexia nervosa contributes significantly to morbidity and mortality among this population of older people.

\section{Conclusion}

Older people may present with anorexia nervosa in medical practice. Awareness of this condition and its association with mood disorders, particularly major depression, is critical to facilitating a timely diagnosis and establishing a treatment plan. We suggest that if physicians suspect an eating disorder in their older patients, they start an early search for appropriate referral services. Furthermore, anorexia nervosa is a significant contributor to morbidity and mortality in affected older people. 
To see this article online, please go to: http://jabfm.org/content/ 30/5/666.full.

\section{References}

1. Beck D, Casper R, Andersen A. Truly late onset of eating disorders: a study of 11 cases averaging 60 years of age at presentation. Int J Eat Disord 1996; 20:389-95.

2. Gadalla TM. Eating disorders and associated psychiatric comorbidity in elderly Canadian women. Arch Womens Ment Health 2008;11:357-62.

3. Scholtz S, Hill LS, Lacey H. Eating disorders in older women: does late onset anorexia nervosa exist? Int J Eat Disord 2010;43:393-7.

4. Mangweth-Matzek B, Rupp CI, Hausmann A, et al. Never too old for eating disorders or body dissatisfaction: a community study of elderly women. Int J Eat Disord 2006;39:583-6.

5. Gagne DA, Von Holle A, Brownley KA, et al. Eating disorder symptoms and weight and shape concerns in a large web-based convenience sample of women ages 50 and above: results of the Gender and Body Image (GABI) study. Int J Eat Disord 2012;45:832-44.

6. Godart NT, Perdereau F, Rein Z, et al. Comorbidity studies of eating disorders and mood disorders. Crit- ical review of the literature. J Affect Disord 2007;97: 37-49.

7. Blinder BJ, Cumella EJ, Sanathara VA. Psychiatric comorbidities of female inpatients with eating disorders. Psychosom Med 2006;68:454-62.

8. Jordan J, Joyce PR, Carter FA, et al. Specific and nonspecific comorbidity in anorexia nervosa. Int J Eat Disord 2008;41:47-56.

9. Lapid MI, Prom MC, Burton MC, McAlpine DE, Sutor B, Rummans TA. Eating disorders in the elderly. Int Psychogeriatr 2010;22:523-36.

10. Hewitt PL, Coren S, Steell GD. Death from anorexia nervosa: age span and sex differences. Aging Ment Health 2001;5:41-6.

11. Diagnostic and Statistical Manual of Mental Disorders, 5th ed. Arlington, VA: American Psychiatric Association; 2013.

12. Mermelstein HT, Basu R. Can you ever be too old to be too thin? Anorexia nervosa in a 92-year old woman. Int J Eat Disord 2001;30:123-6.

13. Gowers SG, Crisp AH. Anorexia nervosa in an 80year-old woman. Br J Psych 1990;157:754-7.

14. Puccio F, Fuller-Tyszkiewicz M, Ong D, Krug I. A Systematic review and meta-analysis on the longitudinal relationship between eating pathology and depression. Int J Eat Disord 2016;49:439-54. 